\title{
Thin-film structures induced by electrostatic field and substrate kinetic constraint
}

\author{
Wei Lü and Dongchoul Kim \\ Department of Mechanical Engineering, University of Michigan, Ann Arbor, Michigan 48109
}

(Received 7 October 2005; accepted 3 March 2006; published online 12 April 2006)

\begin{abstract}
We present a three-dimensional dynamic model for the structural formation of thin polymer films on a substrate subjected to an electrostatic field. The simulations reveal the destabilization of a flat polymer-air interface and the formation of uniform pillars emerging out of the film. We show that this self-assembly process is not solely determined by energetics. The kinetic constraint of the substrate can play an important role. (C) 2006 American Institute of Physics.
\end{abstract}

[DOI: 10.1063/1.2195095]

Recent experiments discovered a process of using electrostatic field to induce self-organized morphological structures. ${ }^{1-3}$ A polymer thin film was spin coated on a substrate, and then heated to above the glass transition temperature. An electric field was applied to the polymer melt, which destabilized a flat polymer-air interface and led to the formation of uniform pillars emerging out of the film. Why does the interface losses stability? What causes the pillars to have a uniform size? Linear or weakly nonlinear perturbation analysis provides clues to the first question. ${ }^{4,5} \mathrm{~A}$ wavy interface reduces the electrostatic energy, but increases the interface energy. The competition determines a critical wavelength. A perturbation with longer wavelength will grow over time. These analyses are valid for the early stage evolution. It is still not clear how the instability may evolve to the final uniform structure. Energetic analysis has shown that the electrostatic energy may not stop the lateral growth. ${ }^{6}$ To illustrate the idea, imagine a two-phase fluid that fills the space between parallel electrodes under a constant voltage. The two phases have different permittivities and form columns that bridge the electrodes. The configuration is analogous to the parallel connection of many capacitors. When the lateral feature size increases, e.g., two columns of the same phase combine into one column, the total interface energy reduces, but the electrostatic energy remains constant. Consequently, the phase size increases laterally to reduce the combined energy. Time permitting, one single column will form. Suo and Liang ${ }^{6}$ postulate that to stop the lateral growth and form stable uniform structures, a special insulating layer may exist on the electrodes.

The dynamics that links the early perturbation to late structure is key to understand the self-assembly behavior. A clear picture of the process requires understanding of the nonlinear evolution regime beyond the perturbation analysis for a flat surface. Particularly, the self-assembly of a thin film is significantly affected by the substrate. The fast growth wave obtained from small perturbation analysis may experience a significant change when it approaches the substrate, and thus may have no direct connection to the size of the late structure. When the film thickness is comparable to or smaller than the fast wavelength, even the early evolution is considerably affected by the substrate. This letter presents a

\footnotetext{
a) Author to whom correspondence should be addressed; electronic mail:
} weilu@umich.edu three-dimensional dynamic model that allows simulation of the entire process. We show that the pillars grow by consuming surrounding film material, leading to decreasing film thickness between pillars. The thin mass transport route and no-slip flow boundary on the substrate may essentially prevent the lateral growth. Uniform structures may emerge due to this kinetic constraint. In such a process the final structure is not determined solely by energy minimization, but by an interplay of energetics and kinetics.

Consider a thin polymer film on a substrate subjected to an electrostatic field. The field is generated by two electrodes parallel to the film, one above the polymer surface and the other underneath the substrate. The system is infinitely large in the lateral direction. When heated, the polymer film may change its morphology via viscous flow and diffusion. The substrate does not evolve. A coordinate system is attached so that the $x_{1}-x_{2}$ plane coincides with the bottom of the substrate. We model the system in a phase field framework. ${ }^{7,8}$ The polymer-air interface is represented by a thin continuous transition region. Similar diffuse interface has also been used for polymer blends. ${ }^{9,10}$ Define a concentration $C$ by the volume fraction of polymer, $C=0$ for air phase and $C=1$ for polymer phase. Regard the concentration as a spatially continuous and time-dependent function $C\left(x_{1}, x_{2}, x_{3}, t\right)$.

The free energy of the system depends on the phase configuration and electric field distribution, namely,

$$
G=\int_{V}\left\{f(C)+\frac{1}{2} h|\nabla C|^{2}-\frac{1}{2} \varepsilon_{0} \varepsilon_{r}(C)|\nabla \phi|^{2}\right\} d V .
$$

The integration extends over the entire volume between the two electrodes. The $f(C)$ term represents the chemical energy, which drives phase separation. We use a double well function $f(C)=f_{0} C^{2}(C-1)^{2}$, where $f_{0}$ is a positive constant. The function has two minima corresponding to the polymer and air phase, respectively. The second term accounts for the interface energy between polymer and air, where $h$ is a material constant. The first two terms are typical in the CahnHilliard model. ${ }^{7}$ The third term represents the electrostatic energy, where $\phi$ is the electric potential, $\varepsilon_{0}$ the vacuum permittivity, and $\varepsilon_{r}$ the dielectric constant of the media. The dielectric constant may be interpolated linearly by those of the polymer and air, namely, $\varepsilon_{r}(C)=\varepsilon_{r}^{\text {polymer }} C+\varepsilon_{r}^{\text {air }}(1-C)$. The specific form is insignificant when the interface region is thin. The electric potential satisfies $\nabla\left(\varepsilon_{r} \nabla \phi\right)=0$ when there 
is no free charge in the dielectric media. This equation combined with boundary conditions gives the electrostatic field for any given concentration distribution. Note that the volume integration in Eq. (1) includes the substrate, whose electric field is affected by the morphology change of the film. For computational convenience, we treat the substrate as a special part of the film which does not evolve, keeps constant $C=1$, and has $\varepsilon_{r}(C)=\varepsilon_{r}^{\text {substrate }}$. Thus Eq. (1) also applies to the substrate since the first two terms disappear automatically. In this way the film and substrate can be treated uniformly. Following experimental observations, ${ }^{2}$ we consider situations that the film wets the substrate to keep their interface intact, i.e., no substrate exposure during evolution. Hence Eq. (1) excludes the nonvarying film-substrate interface energy.

The chemical potential is defined by $\mu=\delta G / \delta C$. When diffusion is the only mass transport mechanism, the diffusion flux is given by $\mathbf{J}=-M \nabla \mu$, where $M$ is mobility. Viscous flow adds a convection term to the flux. We denote the flow velocity by $\mathbf{v}$. The convection flux is given by $C \mathbf{v}$. Note that the film morphology is limited by a slow polymer flow. The air flow can be neglected, i.e., treated as vacuum. We will consider an incompressible flow so that $\nabla \cdot \mathbf{v}=0$. In the concurrent kinetic process, the net flux is the sum of the diffusion flux and the convection flux. This combined with mass conservation gives a convective Cahn-Hilliard equation, namely,

$$
\frac{\partial C}{\partial t}+\mathbf{v} \cdot \nabla C=\nabla(M \nabla \mu) .
$$

With the presence of diffuse interface, a modified NavierStokes equation describes the viscous flow, ${ }^{11}$

$$
\rho\left(\frac{\partial \mathbf{v}}{\partial t}+\mathbf{v} \cdot \nabla \mathbf{v}\right)=-\nabla p+\nabla(\eta \nabla \mathbf{v})+\mu \nabla C .
$$

Here $\rho$ is the density, $\eta$ the viscosity, and $p$ the pressure that enforces the incompressibility constraint. The term $\mu \nabla C$ accounts for the force at the interface. We normalize the governing equations with a characteristic velocity $V_{c}$, length $L_{c}$, and time $t_{c}=L_{c} / V_{c}$. The choice of the magnitudes of the characteristic quantities depends on the physical detail to resolve and the computational convenience. A set of dimensionless equations is given by

$$
\begin{aligned}
& \frac{\partial C}{\partial t}+\mathbf{v} \cdot \nabla C=\frac{1}{\mathrm{Pe}} \nabla(M \nabla \mu), \\
& \mu=4 C^{3}-6 C^{2}+2 C-C^{2} \nabla^{2} C-\frac{1}{2}\left(\Delta \varepsilon_{r}\right)|\nabla \phi|^{2}, \\
& -\nabla p+\nabla(\eta \nabla \mathbf{v})+\frac{1}{\mathrm{Ca}} \mu \nabla C=0 .
\end{aligned}
$$

The mobility $M$ and viscosity $\eta$ are the dimensionless numbers normalized by those of the polymer, $M_{0}$ and $\eta_{0}$. The potential field $\phi$ is normalized by $\phi_{c}=L_{c} \sqrt{f_{0} / \varepsilon_{0}}$. Define $\Delta \varepsilon_{r}=\varepsilon_{r}^{\text {polymer }}-\varepsilon_{r}^{\text {air }}$ in the region above the substrate, and $\Delta \varepsilon_{r}=\varepsilon_{r}^{\text {substrate }}-\varepsilon_{r}^{\text {air }}$ in the substrate. The Péclet number, Pe $=V_{c} L_{c} /\left(M_{0} f_{0}\right)$, reflects the ratio of the diffusive time scale and the convective time scale. The significance of the interface energy is described by the Cahn number, $\mathrm{Ch}$ $=\sqrt{h / f_{0}} / L_{c}$. Equation (6) has neglected the inertial term, i.e.,
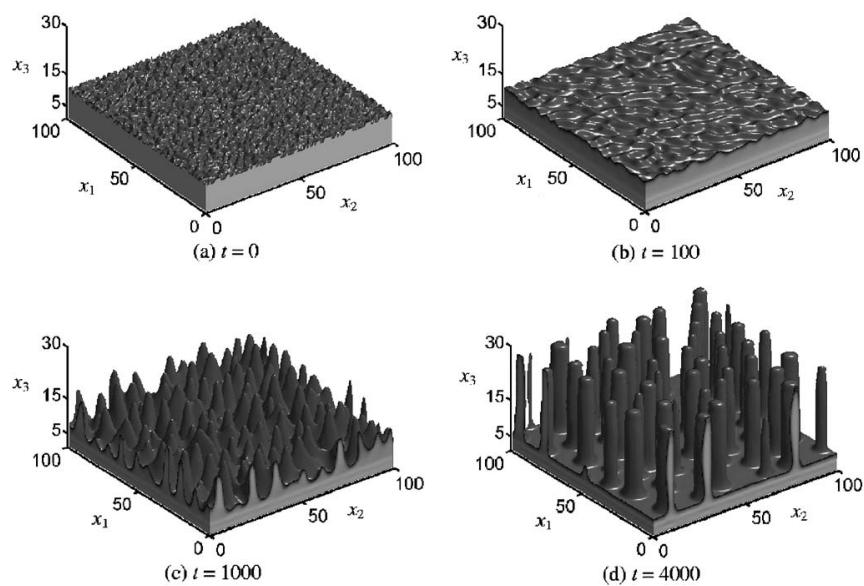

FIG. 1. An evolution sequence of a thin-film with a thickness of 4.5 . (a) $t=0$, (b) $t=100$, (c) $t=1000$, and (d) $t=4000$.

the left hand side of Eq. (3), for the low Reynolds number polymer flow. The capillary number, $\mathrm{Ca}=\eta_{0} V_{c} /\left(L_{c} f_{0}\right)$, affects the relative magnitude of viscous force and interface force.

An efficient semi-implicit Fourier spectral method is used for both high spatial resolution and fast computation. ${ }^{8,12}$ We choose the space between the two electrodes as a calculation domain. The substrate and the polymer film are treated uniformly to avoid the need to explicitly prescribe the flow and diffusion boundary condition at the substrate surface. The uniform treatment also enables application of the efficient spectral method with periodic boundary conditions in three dimensions. We consider the substrate as part of the film, but ensure by kinetics that this part does not flow or diffuse. In other words, we assign $M=0$ and $\eta \gg 1$ in the substrate region so that it keeps $C=1$. Above the substrate, according to the normalization, we have $M=1$ and $\eta=1$. This leads to position dependents $M$ and $\eta$ in Eqs. (4) and (6). We solve Eq. (6) and the incompressibility constraint $\nabla \cdot \mathbf{v}=0$ iteratively in Fourier space with a semi-implicit method to obtain velocity. ${ }^{12}$ In each time step the solutions of the electric field and velocity field are substituted into Eq. (4) to update the concentration field. Numerical details will be presented in a subsequent paper.

The representative results are shown in Figs. 1 and 2. The size of the calculation domain is $100 \times 100 \times 30$. We take typical parameters of polymers: $\varepsilon_{r}^{\text {polymer }}=2.25, \varepsilon_{r}^{\text {air }}=1$, $\varepsilon_{r}^{\text {substrate }}=2.25, \mathrm{Ch}=0.5, \mathrm{Ca}=10$, and $\mathrm{Pe}=10$. Assigning $M$ $=0$ and $\eta=500$ in the substrate region is sufficient to keep it unchanged. The simulations start from random initial conditions, i.e., flat films with small random perturbations. Figure 1 shows an evolution sequence from $t=0$ to $t=4000$ for a film with a thickness of 4.5. The substrate has a thickness of

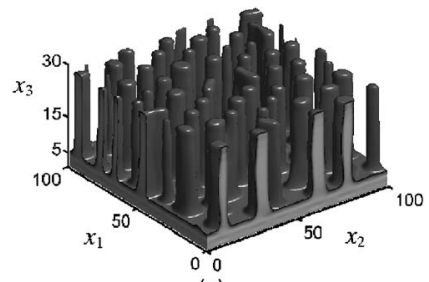

(a)

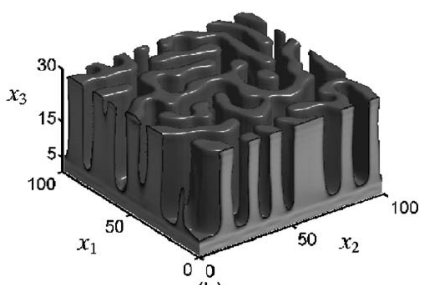

(b)
FIG. 2. Different film thicknesses lead to various morphologies. $t=4000$ thicknesses of 6.5. (a) and 11.5 (b). 
5. A normalized voltage of $U=22$ is applied to the two electrodes, which generates an average field strength of $10^{7}-10^{8} \mathrm{~V} / \mathrm{m}$. Figure 1(a) shows the initial film surface at $t=0$. Surface ripples begin to emerge quickly after a short time of evolution, as shown in Fig. 1(b). The interwoven ripples have different orientations and lengths, but their widths are similar, suggesting a representative size scale. During the growth, longer ripples break into shorter ones. At $t=1000$, the surface evolves into a morphology of many round cones toward the upper electrode, as shown in Fig. 1(c). The cones have a narrow size distribution and are densely packed with their bases in contact. The distance between two neighboring cones is comparable to their size. In this stage, it is relatively easy for cones to combine and expand. First, the cones are short, whose height is comparable to the lateral size. Thus only a small amount of mass transport is needed. Second, there is still sufficient material between the valley of the film surface and the substrate. The viscous flow can happen in a reasonable time despite the no-slip constraint on the substrate surface. The cones continue to grow by absorbing the surrounding material from the valleys and small neighbors. Figure 1(d) shows the formation of quite uniform pillars at $t=4000$, which is consistent with experimental observations. ${ }^{1,2}$ The size of the pillars is similar to that of the cones in Fig. 1(c). However, the distance between pillars is significantly larger than the scale in Fig. 1(c). The simulation suggests that a pillar absorbs the surrounding material for height growth rather than lateral growth. This behavior is understandable since it is the former action that reduces the electrostatic energy. Eventually, the film thickness between pillars becomes so thin that the kinetic constraint of the substrate stops any lateral and height growth after $t=4000$.

The pillar growth in Fig. 1 is fundamentally different from crystal growth in a solution. Crystal growth is a phase transition process limited by diffusion at the growth front. However, pillar growth is limited by mass transport at the base. A pillar grows higher by continuously accumulating underneath surrounding film material, while the growth front, i.e., pillar top, keeps its shape and demonstrates a translational movement. The electrostatic field essentially pulls the pillars towards the upper electrode. A comparison of the early and late stage evolutions, such as Figs. 1(b) and 1(d), suggests a transition from front kinetics to base kinetics. The ripples in Fig. 1(b) have small amplitudes, so that their morphology is directly affected by in situ diffusion and flow. The kinetics happens at the growth front. The pillar growth in Fig. 1(d) is determined by the kinetics at the base. A series of simulations has been performed. It is shown that the pillar size is insensitive to the film thickness, while the in-between distance is significantly affected. A thicker film grows faster and produces more densely packed arrays. Fig- ure 2(a) shows an example for a film with a thickness of 6.5. The comparison with Fig. 1 clearly demonstrates the trend. This trend gradually slows down and approaches the limit of infinite thick film. When the film is thick and the two electrodes are close to each other, the film will continue to supply material to the pillars after they touch the ceiling. This causes pillars to grow laterally and combine each other. An example is shown in Fig. 2(b), which has a thickness of 11.5. This is a relatively thick film, which takes nearly $1 / 2$ of the distance between the top electrode and the substrate. The lateral growth eliminates any initially formed pillars, and leads to a pattern of interwoven stripes. Further evolution far beyond $t=4000$ does not show any significant morphology change. The remaining film between the stripes is so thin that the mass transport essentially stops. The kinetic constraint of the substrate has prevented further lateral growth. Otherwise, as we have observed in the simulations of very thick films, interwoven stripes can continue to grow laterally and combine into one big pillar to minimize the surface energy.

As a potential low-cost and high-throughput nanofabrication approach, self-assembly has significant technical potentials and poses intriguing scientific problems. It is interesting to point out that recently plasma etching of polymers under an applied electric field has produced very similar patterns. ${ }^{13}$ The work here may help to advance the understanding of the mechanism, although plasma etching is more complicated and involves multiple physical and chemical processes. The simulations in this letter reveal rich dynamics and call for further study of these self-assembly processes.

The authors acknowledge financial support from National Science Foundation Career Award No. DMI-0348375.

${ }^{1}$ S. Y. Chou, L. Zhuang, and L. J. Guo, Appl. Phys. Lett. 75, 1004 (1999).

${ }^{2}$ E. Schäffer, T. Thum-Albrecht, and T. P. Russell, Nature (London) 403, 874 (2000); M. D. Morariu, N. E. Voicu, E. Schäffer, Z. Lin, T. P. Russell, and U. Steiner, Nat. Mater. 2, 48 (2003).

${ }^{3}$ D. Salac, W. Lu, C.-W. Wang, and A. M. Sastry, Appl. Phys. Lett. 85, 1161 (2004)

${ }^{4}$ E. Schäffer, T. Thum-Albrecht, T. P. Russell, and U. Steiner, Europhys. Lett. 53, 518 (2001); L. F. Pease III and W. B. Russel, J. Chem. Phys. 118, 3790 (2003)

${ }^{5}$ L. Wu and S. Y. Chou, Appl. Phys. Lett. 82, 3200 (2003).

${ }^{6}$ Z. Suo and J. Liang, Appl. Phys. Lett. 78, 3971 (2001).

${ }^{7}$ J. W. Cahn and J. E. Hilliard, J. Chem. Phys. 28, 258 (1958).

${ }^{8}$ W. Lu and Z. Suo, J. Mech. Phys. Solids 49, 1937 (2001); Phys. Rev. B 65, 085401 (2002); 65, 205418 (2002).

${ }^{9}$ T. Roths, C. Friedrich, M. Marth, and J. Honerkamp, Rheol. Acta 41, 211 (2002).

${ }^{10}$ B. J. Keestra, P. C. J. V. Puyvelde, P. D. Anderson, and H. E. H. Meijer, Phys. Fluids 15, 2567 (2003).

${ }^{11}$ M. E. Gurtin, D. Polignone, and J. Viñals, Math. Models Meth. Appl. Sci. 6, 815 (1996).

${ }^{12}$ J. Zhu, L.-Q. Chen, J. Shen, and V. Tikare, Phys. Rev. E 60, 3564 (1999).

${ }^{13}$ N. D. Tran, N. K. Dutta, and N. R. Choudhury, Thin Solid Films 491, 123 (2005) 